\title{
Los debates políticos como herramienta docente en otros contextos
}

\section{María de Miguel Molina ${ }^{\mathrm{a}}$, Daniel Catalá Pérez ${ }^{\mathrm{b}}$ y Ana Albalat-Mascarell ${ }^{\mathfrak{c}}$}

${ }^{a}$ Departamento de Organización de Empresas, Universitat Politècnica de València, mademi@omp.upv.es, ${ }^{b}$ Departamento de Organización de Empresas, Universitat Politècnica de València, dacapre@ade.upv.es, y ${ }^{\mathrm{c}}$ Departamento de Lingüística Aplicada, Universitat Politècnica de València, analmas@idm.upv.es

\begin{abstract}
At polytechnical universities where our different subjects are offered, analysis of documents related to political discourse is far from frequent. However, this kind of documents may provide valuable information that can be used in Social Science subjects as well as in the teaching of foreign languages at a technical university. In recent years, we have used different political programmes and transcripted debates in order to apply content analysis like an objective tool to search and gather information. Also, we have used content analysis to explore the deployment of linguistic and rhetorical patterns that allow language learners to deal with some key pragmatic skills within a foreign language teaching environment. In this case, we analyse the debates of the last Presidential Elections in the United States of America and their use in the subject Research Methodologies and we compare it with other activities of the subject English for Specific Purposes. With a proper design of the session, we can work on the specific competence of each subject to guide students to apply content analysis to meet their objectives around a hot topic of discussion today.
\end{abstract}

Keywords: political debates, content analysis, discourse analysis, foreign language teaching, instrumental competence, research methodologies.

\footnotetext{
Resumen

En una universidad politécnica como en la que se encuadran nuestras asignaturas, no es habitual el análisis de documentos relacionados con el discurso político. Sin embargo, estos nos proporcionan una información muy valiosa para poder desarrollarse tanto en asignaturas de las Ciencias Sociales como en las ofertadas para conseguir la acreditación del conocimiento de una lengua extranjera en los planes de estudio de titulaciones técnicas. Los programas de los partidos políticos o aquellos debates que han sido transcritos pueden servirnos para utilizar el análisis de contenido bien como una herramienta objetiva de búsqueda de información, bien para identificar y clasificar los recursos lingüisticos y retóricos empleados en dichos contextos y ligados a las competencias pragmáticas cuya adquisición se trabaja en los laboratorios de idiomas. En este caso,
} 
analizamos el uso de los debates de las últimas elecciones a Presidente de los Estados Unidos de América en el ámbito académico de las metodologías de investigación cualitativas y lo comparamos con otras actividades en el ámbito del inglés para fines especificos. Con una planificación adecuada, se puede trabajar la competencia especifica requerida en cada asignatura para que los alumnos conozcan y manejen esta herramienta en torno a un tema de actualidad.

Palabras clave: debates politicos, análisis de contenido, análisis del discurso, competencia instrumental, metodologías de investigación.

\section{Introducción.}

De acuerdo con el proyecto Tuning (European Union, 2007), las "competencias representan una combinación dinámica de conocimiento, comprensión, habilidades y aptitudes". Además, las competencias pueden ser específicas de una asignatura o genéricas (transversales).

Asimismo, dentro de las transversales, encontramos la competencia "instrumental específica" que está íntimamente relacionada con la asignatura que se desarrolla. De acuerdo con la Universitat Politècnica de València (UPV, 2012), "esta competencia hace referencia al uso de las herramientas y tecnologías necesarias para el ejercicio profesional asociado a cada titulación. El estudiante será capaz de identificar las herramientas más adecuadas en cada caso, conociendo sus utilidades y siendo capaz de integrarlas y combinarlas para poder resolver un problema, realizar un proyecto o un experimento".

En los casos que presentamos, se ha trabajado esta competencia para que los alumnos sean capaces de conocer la utilidad del método de "análisis de contenido" y cuándo y cómo aplicarlo.

De acuerdo con Berg y Lune (2014), el análisis de contenido en Ciencias Sociales puede utilizarse tanto en análisis cualitativos como cuantitativos, inductivos (Grounded Theory) o deductivos (comprobar teorías). Va más allá de contar palabras, examinando significados o temas que pueden ser manifiestos o latentes en un texto particular. De este modo, ayuda a los estudiantes a entender la realidad social de una manera científica, así como también a potenciar sus habilidades lingüísticas y pragmáticas en el marco de un tema o contexto discursivo en particular.

Concretamente, en el caso de la enseñanza de idiomas para fines específicos dentro del ámbito universitario, el análisis de contenido puede resultar un instrumento útil para analizar detalladamente transcripciones de discursos auténticos y poner de relieve ciertos características del lenguaje oral que, de otro modo, pasarían inadvertidos para los estudiantes, repercutiendo positivamente en su proceso de aprendizaje. De acuerdo con Chan (2017), el aprendiz de un idioma extranjero, sobre todo aplicado a contextos específicos, agradece el uso de transcripciones de situaciones reales en áreas concretas de trabajo. Además, el análisis de contenido mediante transcripciones puede equipararse a

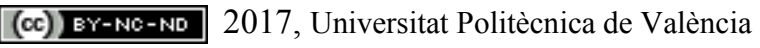


otras herramientas como las WebQuest (Gural y Shulgina, 2015) para fomentar el aprendizaje autónomo y permanente del alumno.

En la actualidad, se dispone en el mercado de algunos software que ayuda a todos estos tipos de análisis como son: VOSviewer, ATLASti, Vantage, etc. En los casos que presentamos, al tratarse de primeras sesiones para los alumnos, no se utilizaron pues se dejaron para sesiones posteriores.

En definitiva, el análisis de contenido se configura, por tanto, como una valiosa herramienta, rigurosa y sencilla, que contribuye a dar respuesta a muchas de las necesidades y los objetivos definidos en un contexto de investigación docente (Ruiz Silva 2004, p. 45). Por ejemplo, Cabrera Ruiz (2009) plantea el análisis de contenido como un método recurrente para la interpretación y evaluación de cualquier tipo de información obtenida en un proceso de investigación educativa; Certad (2015) aplica el análisis de contenido como estrategia evaluativa para un entorno de aprendizaje colaborativo; y Boronat Mundina (2005) lo emplea como metodología para iniciar a los alumnos de postgrado en tareas de investigación. Además, desde un enfoque tal vez más cercano al del presente trabajo, Messinger (2012) introduce a los alumnos de Grado en la utilización de la herramienta a través del análisis de las bandas sonoras de las películas de Harry Potter.

Tanto Messinger (2012) como Boronat Mundina (2005), coinciden en la importancia de escoger un objeto de análisis que resulte atractivo para los alumnos a la hora de iniciarlos en el uso de esta herramienta. En este sentido, se ha considerado que el análisis de un debate político de plena actualidad podría cumplir con este objetivo.

\section{Objetivos.}

Se siguió un método deductivo, pues nuestro primer objetivo era que, basándose en una investigación publicada, se comprobase si esos resultados previos se cumplían o no en un ámbito distinto y en un periodo de tiempo diferente.

Este tipo de estudios comparativos también les ayuda a no perder la mirada crítica ante un documento, sea del tipo que sea, para ir más allá y sacar sus propias conclusiones. De hecho, el análisis de contenido permite construir un nuevo documento distinto al anterior, pero basado en él, más completo, sintético y estructurado, gracias al vínculo que el uso de la herramienta establece entre tres diferentes niveles de lenguaje (Ruiz Silva 2004, p. 46):

- El nivel superficial, formado por el conjunto de información presente en el documento objeto de análisis.

- El nivel analítico, que consiste en la clasificación, ordenamiento de la información y construcción de categorías.

- El nivel interpretativo, en el que los alumnos tratan de comprender el sentido de la información analizada (nivel de superficie), una vez organizada (nivel analítico) y dotándola de un sentido nuevo. 
Asimismo, una vez comprobados dichos resultados, el segundo objetivo era comparar con otras experiencias cómo los patrones lingüístico-discursivos sirven como ejemplo a los alumnos de cómo persuadir y potenciar una imagen de credibilidad y profesionalidad empleando estas estrategias retóricas en el idioma a aprender (en este caso, el inglés).

\section{Desarrollo de la innovación.}

Nuestro punto de partida era el trabajo de Lee y Lim (2016), que se basa en mensajes de redes sociales de Hillary Clinton y Donald Trump publicados durante 2015, para establecer cualidades (traits) y temas (issues) masculinos y femeninos.

Para nuestra práctica en la asignatura de metodologías de investigación cualitativas se eligió el Primer Debate Presidencial Clinton vs. Trump de 2016, emitido en la NBC y transcrito por la revista TIME.

Seguimos los pasos del análisis de contenido descritos por Berg y Lune (2014):

1. Preparación de los datos en texto escrito. No era necesario pues la transcripción ya estaba hecha.

2. Definición de la unidad de análisis (palabra, tema, frase, párrafo). En este caso, primero había que identificar en el trabajo de Lee y Lim (2016) la información.

3. Categorías y esquema de codificación. La información recopilada tenían que clasificarla en: cualidades masculinas, cualidades femeninas, temas masculinos y temas femeninos.

4. Discusión de la codificación por grupos, para dar consistencia al diseño. En esta fase, deben acordarse las categorías y su codificación, para que todo el equipo investigador siga el mismo esquema (Tabla 1).

Tabla 1. Códigos acordados por los equipos

\begin{tabular}{|c|c|}
\hline feminine traits & masculine traits \\
\hline TF1 warm & TM1 strong \\
TF2 caring & TM2 tough \\
TF3 understanding & TM3 assertive \\
TF4 compassionate & TM4 competent \\
TF5 expressive & TM5 instrumental \\
TF6 family-oriented & TM6 achievement-oriented \\
\hline feminine issues & masculine issues \\
\hline IF1 education & IM1 economy \\
IF2 health & IM2 military \\
IF3 human right & IM3 foreign affairs \\
IF4 women's rights & IM4 technology \\
\hline
\end{tabular}

(c) EY-NC-ND 2017, Universitat Politècnica de València 


\begin{tabular}{|c|c|}
\hline IF5 animal right & IM5 science, crime \\
IF6 child care & IM6 terrorism \\
IF7 poverty & IM7 gun problems \\
IF8 arts & \\
IF9 environment & \\
IF10 social welfare & \\
\hline
\end{tabular}

5. Con esta clasificación ya se puede codificar todo el texto. Conforme se va leyendo, se apuntan los códigos. Si fuesen muchos textos, podríamos utilizar un software que nos facilitase este trabajo.

6. Se re-comprueba la consistencia, por ejemplo comparando entre dos compañeros si se ha codificado la misma información.

\section{Resultados.}

El siguiente paso es escribir los resultados del proceso de codificación, interpretando y justificando el porqué de dichos resultados. Al tratarse de un estudio cualitativo, interpretación y teoría deben ir de la mano para dar consistencia científica a los resultados.

Por ejemplo, determinados resultados pueden ir acompañados de extractos del texto entrecomillados que justifiquen esa interpretación.

Se les pidió a los estudiantes que los resultados se mostrasen comparando a ambos candidatos, y posteriormente cada grupo expuso sus conclusiones al resto de la clase.

Los resultados obtenidos mostraron que, en este debate, Clinton expresaba cualidades y temas femeninos en su mayoría, aunque en ocasiones también citaba temas masculinos. Por otro lado, Trump solo mostraba cualidades masculinas y todos sus temas también eran masculinos.

Los resultados no solo ayudaron a entender y practicar el análisis de contenido, sino también a debatir sobre su utilidad. En este caso, los dos candidatos podrían derivar sus próximos discursos o debates en cualidades o temas que no han mencionado, para llegar a un mayor número de electores. Asimismo, los debates muestran a los candidatos de una manera más clara que las redes sociales, que era la base del trabajo de Lee y Lim (2016), que pueden ser manejadas por otras personas contratadas para ello (community managers).

Al comparar esta práctica de la asignatura de Metodologías de investigación, con otras actividades realizadas en Inglés para fines específicos, vemos que el análisis de discursos políticos también puede servir para detectar estrategias lingüísticas y retóricas utilizadas en cada unidad de análisis codificada de acuerdo a las dicotomías descritas. En el caso que se compara, por ejemplo, serían cualidades masculinas y cualidades femeninas, temas masculinos y temas femeninos. 
Así, al utilizar recursos retóricos, los candidatos pueden emplear matizadores, realzadores y marcadores de actitud muy diferentes (Hyland, 2015) en función de las cualidades y temas tratados. De este modo se pueden observar diferencias significativas en cuanto a las estrategias metadiscursivas preferidas por los candidatos.

Sin embargo, si no se observan grandes diferencias en lo que respecta al número de elementos retóricos empleados según el tema y la cualidad tratada, los estudiantes solo tienen en cuenta las variables cualitativas a la hora de tomar ejemplo de las transcripciones para trabajar los recursos pragmáticos y discursivos del idioma.

\section{Conclusiones.}

El análisis de contenido de temas de actualidad nos ayuda no solo a trabajar la competencia instrumental específica (uso de metodologías de investigación o estrategias pragmáticas orales en una lengua extranjera) sino otras como el trabajo en equipo o el pensamiento crítico. Es decir, más allá de la indudable aplicabilidad de la técnica en el campo de la investigación social (cuantitativa y/o cualitativa), el análisis de contenido ayuda al alumno a organizar su pensamiento, configurándose, por tanto, como una herramienta que promueve y facilita el proceso de aprendizaje.

Se ha combinado el uso de un trabajo científico junto a un texto divulgativo, de manera que en este caso también se perciba la utilización del método científico en diferentes profesiones y no meramente en el ámbito académico. Se amplia, así, el horizonte de los alumnos al descubrir los diferentes contenidos que pueden ser objeto de análisis y se verifica y enfatiza la proyección práctica que puede tener, en concreto, esta herramienta.

\section{Referencias}

BERG, B.L. y LUNE, H. (2014). Qualitative research methods for the social sciences. Harlow: Pearson Education.

BORONAT MUNDINA, J. (2005). "Análisis de contenido. Posibilidades de aplicación en la investigación educativa" en Revista Interuniversitaria de Formación del Profesorado, Volume 19, Issue 2, pp. 157-174. < http://www.redalyc.org/articulo.oa?id=27419209> [Consulta: $29 \mathrm{de}$ marzo de 2017]

CABRERA RUIZ, I. (2009). "El análisis de contenido en la investigación educativa: propuesta de fases y procedimientos para la etapa de evaluación de la información" en Pedagogía Universitaria, Volume 14, Issue 3, pp. 71-93. ISSN 1609-4808. $<$ http://cvi.mes.edu.cu/peduniv/index.php/peduniv/article/view/501> [Consulta: 29 de marzo de 2017]

CERTAD, P.A. (2015). "Diseño de un instrumento para la evaluación de entornos de aprendizaje colaborativo" en Tendencias emergentes en evaluación formativa y compartida en docencia: El papel de las nuevas tecnologias, Santander: Ed. Universidad de Cantabria, pp. 325-354.

CHAN, C. (2017). "Investigating a research-informed teaching idea: The use of transcripts of authentic workplace talk in the teaching of spoken business English" en English for Specific

(c)) BY-NC-ND 2017, Universitat Politècnica de València 
Purposes, Volume 47, pp. 72-89. <http://dx.doi.org/10.1016/j.esp.2016.12.002> [Consulta: 29 de marzo de 2017]

EUROPEAN UNION (2007). Tuning Educational Structures in Europe (competences). $<$ http://www.unideusto.org/tuningeu/competences.html> [Consulta: 23 de marzo de 2017]

GURAL, S. y SHULGINA, E. (2015) "Socio-Cognitive Aspects in Teaching Foreign Language Discourse to University Students" en Procedia - Social and Behavioral Sciences, Volume 200, pp. 3-10. <https://doi.org/10.1016/j.sbspro.2015.08.002> [Consulta: 29 de marzo de 2017]

HYLAND, K. (2015). "Metadiscourse" en The International Encyclopedia of Language and Social Interaction, Volume 1, pp. 1-11. <http://dx.doi.org/10.1002/9781118611463.wbielsi003> [Consulta: 29 de marzo de 2017]

LEE, J. y LIM, Y. (2016). "Gendered campaign tweets: The cases of Hillary Clinton and Donald Trump" en Public Relations Review, Volume 42, Issue 5, pp. 849-855. $<$ http://dx.doi.org/10.1016/j.pubrev.2016.07.004> [Consulta: 23 de marzo de 2017]

MESSINGER, A.M. (2012). "Teaching Content Analysis through Harry Potter" en Teaching Sociology, Volume 40, Issue 4, pp. $360-367 \quad<$ http://journals.sagepub.com/doi/abs/10.1177/0092055X12445461> [Consulta: 29 de marzo de 2017]

RUIZ SILVA, A. (2004). "Texto, testimonio y metatexto : el análisis de contenido en la investigación en educación” en La práctica investigativa en ciencias sociales, Bogotá: Universidad Pedagógica Nacional UPN, pp. 44-59.

UNIVERSITAT POLITÈCNICA DE VALÈNCIA, UPV (2012). Competencias Transversales, competencia instrumental especifica. $<$ http://www.upv.es/contenidos/COMPTRAN/info/955170normalc.html> [Consulta: 23 de marzo de 2017] 